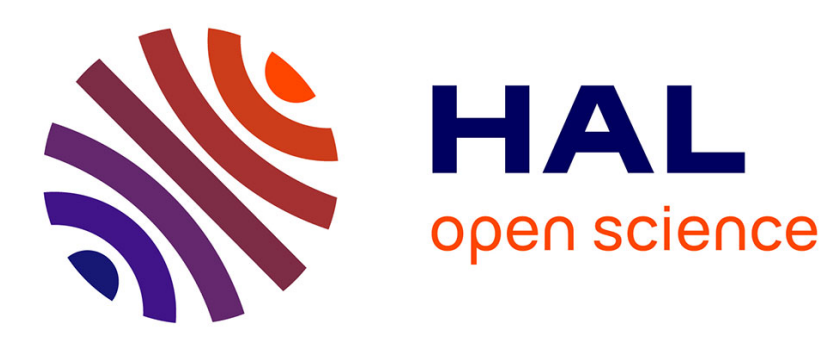

\title{
On the order of the spin glass transitions in mean field theory
}

\author{
G. Toulouse, M. Gabay, T.C. Lubensky, J. Vannimenus
}

\section{To cite this version:}

G. Toulouse, M. Gabay, T.C. Lubensky, J. Vannimenus. On the order of the spin glass transitions in mean field theory. Journal de Physique Lettres, 1982, 43 (4), pp.109-113. 10.1051/jphyslet:01982004304010900 . jpa-00232017

\section{HAL Id: jpa-00232017 https://hal.science/jpa-00232017}

Submitted on 1 Jan 1982

HAL is a multi-disciplinary open access archive for the deposit and dissemination of scientific research documents, whether they are published or not. The documents may come from teaching and research institutions in France or abroad, or from public or private research centers.
L'archive ouverte pluridisciplinaire HAL, est destinée au dépôt et à la diffusion de documents scientifiques de niveau recherche, publiés ou non, émanant des établissements d'enseignement et de recherche français ou étrangers, des laboratoires publics ou privés. 
Classification

Physics Abstracts

$05.50-75.50 \mathrm{~K}$

\title{
On the order of the spin glass transitions in mean field theory
}

\author{
G. Toulouse, M. Gabay, T. C. Lubensky and J. Vannimenus \\ Laboratoire de Physique de l'Ecole Normale Supérieure, \\ 24, rue Lhomond, 75231 Paris Cedex 05, France
}

(Reçu le 13 novembre 1981, accepté le 16 décembre 1981)

\begin{abstract}
Résumé. - A la lumière de ce qu'on sait maintenant sur la théorie de champ moyen,-les transitions verre de spin, en présence d'un champ magnétique, y apparaissent comme étant du troisième ordre, au sens d'Ehrenfest.
\end{abstract}

\begin{abstract}
We present evidence that the mean field transitions in spin glasses in the presence of a magnetic field are third order in the Ehrenfest sense.
\end{abstract}

In the infinite-ranged SK model [1] (which defines for us mean field theory) a spin glass transition occurs, in zero magnetic field, which is third order in the Ehrenfest sense, with cusps in the specific heat and in the magnetic susceptibility. Under finite magnetic field, the transition point splits up, yielding two transition lines in the phase diagram $(H:$ magnetic field, $T:$ temperature) for classical vector spins $[2,3]$. As $T$ decreases, for a given $H$, the first transition line which is met corresponds to freezing of the transverse spin components; the second is due to the onset of replica symmetry breaking (Fig. 1). For Ising spins, obviously, only the latter is found.

This letter reconsiders the nature of these spin glass transitions in finite field. Some previous views $[4,5]$ are discussed and corrected. Present evidence favours a third order character for both transitions.

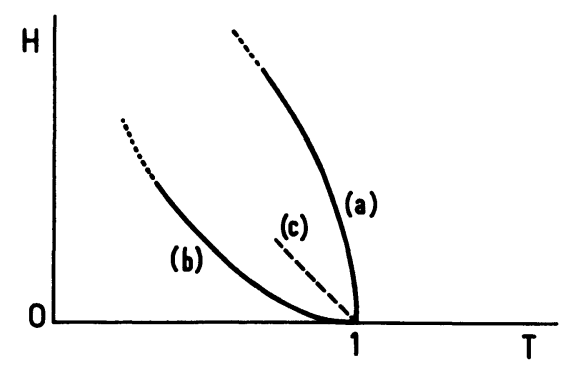

Fig. 1. - Phase diagram of the infinite-ranged model for classical vector spins ( $H$ magnetic field, $T$ temperature). Line (a) corresponds to the transverse freezing transition, line (b) to the onset of replica symmetry breaking. The dashed line (c) indicates a crossover region, on each side of which some quantities have different analytic expansions. 
1. Transverse freezing temperature. - This transition is the simpler, because an ordinary order parameter can be defined, namely the transverse Edwards-Anderson order parameter $q_{\mathrm{T}}$. Analytic results are obtained by expansions around the point $\left(H=0, T_{\mathrm{c}}=1\right)$. These expansions must be handled with care because the relative importance of the various terms depends sensitively on the specific region of the $H-T$ plane in the vicinity of this point.

Up to lowest order in $H$, the transverse freezing transition temperature $T_{\mathrm{c}}$ is found to be :

$$
T_{\mathrm{c}_{2}}=\left[\left\langle S_{x}^{2}\right\rangle^{2}\right]^{1 / 2}=1-\frac{H^{2}}{4} \frac{m^{2}+4 m+2}{(m+2)^{2}}+O\left(H^{4}\right),
$$

where the index $x$ denotes any transverse direction with respect to the applied field. Note that the coefficient of $H^{2}$ in (1) differs from the expressions presented in [2,3], due to an inadvertant neglect therein of a higher order term which also contributes. Formulae (11) and (16) in [2], formulae (6) and (10) in [3] should be corrected accordingly.

Below the transition, it is found that, under small field $H$,

$$
q_{\mathrm{T}}=A\left(T_{\mathrm{c}_{2}}-T\right)+0\left[\left(T_{\mathrm{c}_{2}}-T\right)^{2}\right],
$$

where $A \simeq 1+O\left(H^{2}\right)$. As a consequence, the free energy increase below the transition is

$$
\Delta F \simeq \frac{(m-1)}{6} q_{\mathrm{T}}^{3} \simeq \frac{(m-1)}{6} \tau^{3}
$$

Note that, in strictly zero field,

$$
\Delta F \simeq \frac{m}{6} q^{3} \simeq \frac{m}{6} \tau^{3}
$$

because, in this isotropic case, all spin components contribute equally. Thus the limiting value for $\Delta F$, when $H \rightarrow 0$, is not equal to its value at the limit. This is due to the existence of a crossover region $H \simeq 1-T$ in the phase diagram (line (c) in Fig. 1) which prevents expansions on one side to be validly continued to the other side. This phenomenon will be found repeatedly and must be treated properly.

Formula (4) implies the existence of a cusp in the specific heat at $T_{\mathrm{c}}$ :

$$
\frac{\partial}{\partial T}\left(\frac{\Delta C}{T}\right) \simeq m-1, \text { for } H \text { small }
$$

Repeated use of Clausius-Clapeyron formulae along the transition line yields :

$$
\frac{\partial}{\partial T}\left(\frac{\Delta C}{T}\right)=-\frac{\partial}{\partial H}\left(\frac{\Delta C}{T}\right) \cdot \frac{\mathrm{d} H}{\mathrm{~d} T_{\mathrm{c}_{2}}}=\frac{\partial \Delta x}{\partial T} \cdot\left(\frac{\mathrm{d} H}{\mathrm{~d} T_{\mathrm{c}_{2}}}\right)^{2}=-\frac{\partial \Delta x}{\partial H} \cdot\left(\frac{\mathrm{d} H}{\mathrm{~d} T_{\mathrm{c}_{2}}}\right)^{3},
$$

where the value of $\frac{\mathrm{d} H}{\mathrm{~d} T_{\mathrm{c}_{2}}}$ can be obtained from (1), for $H$ small.

As a consequence, the longitudinal magnetization picks up a quadratic increment below the transition (Figs. 2 and 3) :

$$
\Delta M \simeq \frac{(m-1)}{2}\left(T-T_{\mathrm{c}_{2}}\right)^{2} \frac{H_{\mathrm{c}_{2}}}{2} \cdot \frac{m^{2}+4 m+2}{(m+2)^{2}},
$$




$$
\Delta M \simeq \frac{(m-1)}{2}\left(H-H_{\mathrm{c}_{2}}\right)^{2} \frac{H_{\mathrm{c}_{2}}^{3}}{8} \cdot \frac{\left(m^{2}+4 m+2\right)^{3}}{(m+2)^{6}},
$$

for $T_{\mathrm{c}_{2}} \simeq 1, H_{\mathrm{c}_{2}}$ small

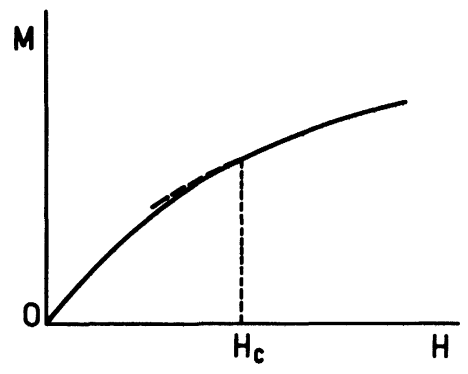

Fig. 2. - Longitudinal magnetization $M$ versus field $H$ at a given temperature $T$. Below the transition at $H_{\mathrm{c}}, M$ has a quadratic increment in $H-H_{\mathrm{c}}$ (dashed line).

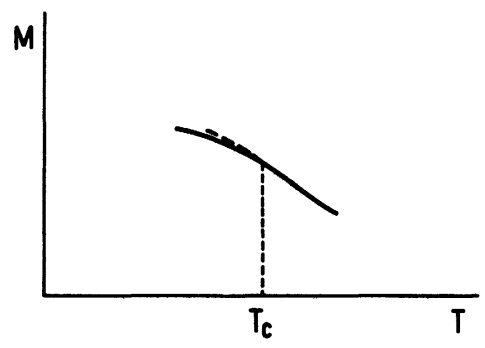

Fig. 3. - Longitudinal magnetization $M$ versus temperature $T$, in a given field $H$. Below the transition at $T_{\mathrm{c}}, M$ has a quadratic increment in $T-T_{\mathrm{c}}$ (dashed line).

That this increment must be positive just below the transition is a necessary consequence of the maximization rule for the free energy as a function of the order parameter $q$, and of the relation :

$$
M=-\frac{\partial F}{\partial H}
$$

2. Replica symmetry breaking transition. - The nature of this second transition is subtler ; in particular, it is predicted that the usual order parameter is replaced by an " order function " [6]. Despite this complication, some general arguments can still be used.

The last argument of the preceding section applies here also. Just below the transition, the longitudinal magnetization must increase above the analytic continuation of the higher temperature phase expression :

$$
\Delta M>0 \text {. }
$$

This implies that the projection hypothesis [4] cannot be strictly valid. The discrepancy may be tiny because the instability line is quite close to the locus of maxima for the paramagnetic $M(T)$, at least for Ising spins [5]. However, there is also a question of principle : the projection hypothesis implied a second order transition. Rule (10) would rather suggest a third order transition. 
In [4], an argument based on Clausius-Clapeyron relations was given against a third order transition. It was said that, with this assumption, the relation

$$
\frac{\partial}{\partial T}\left(\frac{\Delta C}{T}\right)=\frac{\partial}{\partial T} \Delta \chi \cdot\left(\frac{\mathrm{d} H_{\mathrm{c}}}{\mathrm{d} T}\right)^{2}
$$

would have an unacceptable limit on the zero-field axis. There is a snag in this argument, unfortunately. The snag is that the limiting value differs from the value at the limit, again. For any finite $H$, one is talking about quantities taken on the left of line (c) in figure 1 , whereas for $H$ strictly zero, this is not the case.

This objection being cleared up, it is now possible to give an argument in favour of a third order transition. Various approaches, while differing in higher order terms, agree in the first terms of an expansion of the magnetization in powers of the magnetic field, for $T \lesssim 1$ (we are talking now about Ising spins) :

$$
\frac{M}{H} \simeq 1-\frac{\tau^{2}}{3}-\frac{H^{2}}{2 \tau}, \quad \tau=1-T,
$$

for the paramagnetic expression, and

$$
\frac{M}{H} \simeq 1-\left(\frac{3}{4}\right)^{2 / 3} H^{4 / 3}
$$

for the spin glass expression, i.e. the correct one on the low temperature side of the instability line.

Expressions (12) and (13) imply that the magnetization and the susceptibility are continuous on the instability line whose equation is :

$$
H^{2} \simeq \frac{4}{3} \tau^{3}
$$

However, there is a cusp in the susceptibility of the form :

$$
\frac{\partial \Delta \chi}{\partial H} \simeq \frac{2}{3}\left(\frac{4}{3}\right)^{1 / 2} \tau^{1 / 2},
$$

for $\tau$ small.

Relations (6) can be used again. It is worth noticing that at both the transverse freezing and replica symmetry breaking transitions, $\Delta M$ is positive even though other aspects (remanence effects, etc.) of the transitions are different.

For classical vector spins, relations (12), (13), (15) become respectively :

$$
\begin{aligned}
\frac{M}{H} & \simeq 1-\frac{\tau^{2}}{m+2}-\frac{H^{2}}{2 \tau}, \\
\frac{M}{H} & \simeq 1-\frac{3}{2.2[(m+2)]^{1 / 3}} H^{4 / 3}, \\
\frac{\partial \Delta \chi}{\partial H} & \simeq \frac{4}{3(m+2)^{1 / 2}} \tau^{1 / 2},
\end{aligned}
$$

where $m$ is the number of spin components. For $m=1$, the Ising case is recovered. 
3. Conclusion. - It is plausible that the spin glass transitions, in finite field, are third order, with a quadratic increment of the magnetization below the transitions, as illustrated in figures 2 and 3. This implies that the projection hypothesis for the replica symmetry broken phase is only an approximation, though apparently a rather good one. As for the transverse freezing transition, our results are a contribution toward its proper experimental identification.

Finally, it must be kept in mind that a change of order along the transition lines, in higher field, cannot be ruled out, at this stage of the theory.

Acknowledgments. - Stimulating discussions or correspondence with P. Monod, R. Tournier, A. Bray, M. Hardiman, U. Krey, A. Malozemoff are gratefully acknowledged.

Note added in proof. - It has recently been found that, in the vector case, replica symmetry instability in the transverse components occurs as soon as transverse freezing sets in. This fact puts the existence of a second transition into question. However the relevance of the remarks made in the present paper, concerning the nature of the transition first met from the paramagnetic phase (in the Ising as well as in the vector case), is not affected by these considerations.

\section{References}

[1] Sherrington, D., KirkPatrick, S., Phys. Rev. Lett. 32 (1975) 1792.

[2] Toulouse, G., Gabay, M., J. Physique Lett. 42 (1981) L-103.

[3] Gabay, M., Toulouse, G., Phys. Rev. Lett. 47 (1981) L-201.

[4] Parisi, G., Toulouse, G., J. Physique Lett. 41 (1980) L-361.

[5] Vannimenus, J., Toulouse, G., Parisi, G., J. Physique 42 (1981) 565.

[6] PARISI, G., Phys. Rep. 67 (1980) 25, and references therein. 